\title{
Aberrant expression of microRNA induced by high fructose diet: Implications in the pathogenesis of hyperlipidemia and hepatic insulin resistance
}

Neetu Sud ${ }^{1}$, Hanyuan Zhang ${ }^{2},{\text { Kaichao } \text { Pan }^{1}, \text { Xiao Cheng }}^{1}$, Juan Cui ${ }^{2}$ and Qiaozhu Su ${ }^{1 *}$

${ }^{1}$ The Department of Nutrition and Health Sciences, University of Nebraska-Lincoln,

Lincoln, NE USA 68583-0806

${ }^{2}$ Department of Computer Science \& Engineering, University of Nebraska-Lincoln

Short title: Fructose induced microRNAs in the pathogenesis of metabolic disorders

\section{To whom correspondence should be addressed:}

Dr. Qiaozhu Su

316F Leverton Hall

University of Nebraska-Lincoln

Lincoln, NE 68583-0806

E-mail: qsu2@unl.edu

Phone: +1 4024724038

Fax: +1 4024721587

Email: qsu2@unl.edu 


\begin{abstract}
Fructose is a highly lipogenic sugar which can alter energy metabolism and trigger metabolic disorders. In the current study, microRNAs (miRNAs) altered by a high-fructose diet were comprehensively explored to elucidate their significance in the pathogenesis of chronic metabolic disorders. miRNA expression profiling using small non-coding RNA sequencing revealed that 19 miRNAs were significantly upregulated and 26 were downregulated in the livers of high-fructosefed mice compared to chow-fed mice. Computational prediction and functional analysis identified ten miRNAs, miR-19b-3p, miR-101a-3p, miR-30a-5p, miR-223-3p, miR-378a-3p, miR-33-5p, miR-145a-3p, miR-128-3p, miR-125b-5p and miR-582-3p, assembled as a regulatory network to potentially target key genes in lipid and lipoprotein metabolism and insulin signaling at multiple levels. qRT-PCR analysis of their potential target genes [IRS-1, FOXO1, SREBP-1c/2, ChREBP, insulin induced gene-1 (Insig-1)/gene-2 (Insig-2), microsomal triglyceride transfer protein (MTTP) and apolipoprotein B (apoB)] demonstrated that fructose induced alterations of miRNAs were also reflected in mRNA expression profiles of their target genes. Moreover, the miRNA profile induced by high-fructose diet differed from that induced by high-fat diet, indicating that miRNAs mediate distinct pathogenic mechanisms in dietary-induced metabolic disorders. This study presents a comprehensive analysis of a new set of hepatic miRNAs, which were altered by high-fructose diet and provides novel insights into the interaction between miRNAs and their target genes in the development of metabolic syndrome.
\end{abstract}

Key words: Fructose, microRNAs, energy metabolic pathways, high-fat diet, metabolic syndrome 


\section{INTRODUCTION}

Fructose is a monosaccharide abundant in many fruits and vegetables, honey, high fructose corn syrup, soft drinks and prepackaged foods. It was initially considered a beneficial sweetener for diabetic patients due to its low glycemic index compared to glucose, however, recently it has been shown that overconsumption of fructose can be pathogenic. Studies have provided strong evidence of its adverse effects on human and animal health [1]. Fructose is predominantly and rapidly metabolized in the liver where it promotes de novo lipogenesis [1].

Fructose consumption has been shown to enhance the risk of metabolic syndrome, including obesity and insulin resistance and type 2 diabetes (T2D) by accelerating de novo lipogenesis and increasing very low density lipoprotein (VLDL) biogenesis in humans and animal models $[1,2]$. Fructose overconsumption has been reported to cause adverse metabolic effects such as increased visceral and intrahepatic fat accumulation, which are known to promote the development of nonalcoholic fatty liver disease (NAFLD), a highly prevalent liver disease which is linked with metabolic disorders such as obesity, T2D, hypertension and cardiometabolic disease [1]. Although ample research, including epidemiological studies, has described the harmful effects of fructose on human and animal health, the underlying molecular processes behind its lipogenic effects are still poorly understood.

Micro RNAs (miRNAs) are small noncoding RNA ( 22 nucleotide long) that regulate gene expression and function. They bind to the 3'-UTR or 5'-UTR of target mRNA and either suppress protein translation or promote mRNA degradation [3]. More importantly, it has been shown that a single miRNA has the potential to target multiple mRNAs, and a single mRNA can be targeted by multiple miRNAs, which in turn enables them to regulate several groups of mRNAs within a signaling pathway or network and generate a powerful impact on diverse cellular processes [4]. Thus understanding how dietary factors such as fructose and fat can alter miRNA expression and characterizing the diet-induced changes in expression of miRNA and 
their target mRNA is key to understanding the molecular mechanisms underlying the metabolic syndrome.

miRNAs induced by high fat diet (HFD) have drawn considerable attention because of their critical roles in the pathogenesis of metabolic disorders [5]. However, the impact of high fructose diet on hepatic miRNA expression profiles and the putative role of these miRNAs in fructose-induced hyperlipidemia and insulin resistance are not fully understood. In this study, we identified a new set of hepatic miRNA whose expression profiles were significantly altered by high fructose diet using small RNA deep sequencing, followed by in-depth bioinformatics analysis. Subsequent target prediction and pathway functional enrichment analysis further identified the targeted mRNAs of these miRNAs that assembled as hubs which regulate multiple key metabolic pathways that are associated with the onset of diet-induced metabolic disorders. We further compared the miRNA profiles induced by HFD with those induced by high fructose diet and discovered that their miRNA expression profiles were different with certain miRNAs that were upregulated by fructose but downregulated by HFD or vice versa. This may suggest that fructose and HFD induced metabolic disorders via different pathogenic pathways. In summary, our study provides novel mechanistic insights into the fructose induced hyperlipidemia and insulin resistance by identifying a novel miRNA network and their interaction with the key genes in lipid and carbohydrate metabolic signaling.

\section{MATERIALS AND METHODS}

\subsection{Animal protocols}

All animal experiments were approved by the University of Nebraska-Lincoln Institutional Animal Care and Use Committee

\begin{tabular}{l}
\hline Table 1. Detailed composition of the diets in this study \\
\begin{tabular}{||l|l|l|}
\hline Ingredients (\% mass) & Chow & Fructose \\
\hline Protein & 16.4 & 22.21 \\
\hline Fat & 4 & 6.0 \\
\hline Starch and fibers & 52.0 & Trace \\
\hline Fructose & Trace & 60.0 \\
\hline Neutral Detergent Fiber & 15.2 & 7.09 \\
\hline Calories from Protein (\%) & 22 & 21.77 \\
\hline Calories from Fat (\%) & 12 & 14.77 \\
\hline Calories from Carbohydrate (\%) & 66 & 62.37 \\
\hline
\end{tabular}
\end{tabular}
and were carried out under the institutional guidelines for ethical animal use. Male mice 
$(\mathrm{C} 57 \mathrm{BL} / 6 \mathrm{~J})$ at 12 weeks of age were purchased from Jackson Laboratory (Bar Harbor, Maine, USA). MiR-378a-depelted mice (miR-378-KO) mice and their WT littermates are a kind gift from Dr. Vincent Giguere (McGill University, Montreal, Canada). Animals were housed on alternating $12 \mathrm{~h}$ light and dark cycles with free access to food and water. After a week of acclimatization, two groups of mice ( $n=6 /$ per group) were fed either chow (Envigo, Madison, Wisconsin. ID: 2016) or a high-fructose diet (60\% fructose, ID: 161.506 Dyets, Bethlehem, PA) for 4 weeks. Dietary compositions are provided in Table 1 . Food intake and body weight were monitored every three days. Intraperitoneal (IP) glucose tolerance tests (IPGTTs) were performed weekly. Briefly, mice were fasted for $12 \mathrm{~h}$ and then received glucose $(2 \mathrm{~g} / \mathrm{kg}, \mathrm{IP})$ and blood glucose was measured via tail vein bleeding using a handheld glucometer (Contour/Next, Bayer). At the end of the feeding trial, mice were fasted for 12 hours and anesthetized using isoflurane and livers were excised, frozen in liquid nitrogen and stored at $-80^{\circ} \mathrm{C}$ for further analysis.

\subsection{Cell culture, transfection and treatments}

The rat hepatoma cell line McA-RH7777 (McA) was obtained from America Type Culture Collection (ATCC, Manassas, VI) and maintained in DMEM containing 10\% FBS, $4.5 \mathrm{~g} / \mathrm{L}$ glucose and $0.5 \%$ penicillin/streptomycin. Cells were grown in T75 flasks at $37^{\circ} \mathrm{C}, 5 \% \mathrm{CO} 2$, with the media changed every other day and cells passaged every $3-4$ days. For transfection, the cells were seeded onto 6-well plates $\left(0.3 \times 10^{6}\right)$ and maintained in DMEM containing $4.5 \mathrm{~g} / \mathrm{L}$ glucose. When the cells reached $60 \sim 70 \%$ confluence, cells were transfected with $1.0 \mu \mathrm{g}$ of plasmid cDNA encompassed either a GFP reading frame or miR-125b-5p (Vigene Biosciences, Rockville, MD) by Lipofectamine 2000. $24 \mathrm{~h}$ after transfection, the cells were treated with palmitate (100 $\mu \mathrm{M})$ in DMEM (4.5g/L) without FBS for additional $24 \mathrm{~h}$. Total RNAs were collected at the end points of each treatment as described below. 


\section{3 miRNA extraction and miRNA sequencing profiling}

Total RNA, including miRNA, was isolated from the chow and fructose-fed mouse livers using miRNeasy Mini Kit (Qiagen) following the manufacturer's instructions. The miRNA profiling was conducted using Illimina HiSeq 2500 sequencer by Genomics and Microarray Core in UT Southwestern Medical Center (Dallas, Texas). Libraries were prepared using Illumina TruSeq Small RNA Sample preparation kit (Illumina, USA). Six replicates were sequenced for each treatment.

\subsection{RNA isolation, reverse transcription and qRT-PCR}

Total RNA was isolated from tissue using TRIzol (Life Technologies, Grand Island, NY). RNA integrity was confirmed using a NanoDrop 2000 (Wilmington, DE). First strand cDNA was synthesized with oligo (dT) and random primers using a High-Capacity cDNA Reverse Transcription Kit with RNase Inhibitor (Life Technologies). Quantification of gene expression was performed on Applied Biosystems real-time-PCR system StepOnePlus. Relative induction of gene mRNA expression was calculated using the expression of 18 s for normalization. Sequences of forward and reverse primers (5'to $3^{\prime}$ ) used in this study are shown in Supplementary Table 1.

\subsection{MiRNA Sequencing data Analysis}

For bioinformatics analysis, the CAP-miSeq (http://bioinformaticstools.mayo.edu/research/cap-mirseq) was applied to identify mouse miRNAs and calculate their expression [6]. The miRBase (Version 21) was used as reference library (http://www.mirbase.org/ ftp://mirbase.org/pub/mirbase/CURRENT/README). We have carefully filtered out the low quality reads and strictly mapped the qualified reads to all known mature sequences, precursor sequences and the mouse genomes. The differentially expressed miRNA between fructose and chow fed mice were analyzed using the R package, edgeR and only 
those miRNAs with p-value $<0.05$ were considered significantly differential [7]. Hierarchical clustering (http://bonsai.hgc.jp/ mdehoon/software/cluster/software.htm) was performed and a heat map was generated to show the differential gene patterns where green represented downregulation while red represented upregulation compared to the chow-fed controls.

\subsection{Target prediction and functional analysis}

As the functions of miRNA can be inferred based on its gene targets, we extracted the known miRNA gene targets from miRTarBase (http://mirtarbase.mbc.nctu.edu.tw) and [8], DIANATarbase and other softwares of Diana tools (http://diana.imis.athenainnovation.gr/DianaTools/index.php?r=site/page \&view=software) and also predicted new targets using MiRanda (www.microrna.org) and TargetScan (www.targetscan.org). The gene interaction network was generated through Cytoscape [9].

\subsection{Statistical analyses}

Statistical analysis of Real time PCR data was done with GraphPad Prism 6 (La Jolla, CA, USA) using Student's t-test. All results are presented as means \pm SEM. Asterisks (*) indicate statistically significant differences of $\mathrm{P}<0.05$ compared to controls. 


\section{RESULTS}

3.1 High fructose feeding induces insulin resistance which is accompanied with aberrant expression of miRNAs in mouse livers.

To establish an insulin resistant animal model, two groups of C57BL/6J mice ( $\mathrm{n}=6 /$ group) were fed either chow or a high fructose diet (60\% caloric from fructose). Caloric intake was monitored daily and was found to be comparable between chow and fructose-fed mice (Suppl. Figure 1A). Body weights were comparable between chow and fructose-fed mice during the first two weeks of feeding but were significantly increased in the fructose-fed mice from week 3 (Suppl. Figure 1B). At week 4 of high fructose feeding, plasma triglycerides and cholesterol were significantly higher in the fructose-fed mice compared to the chow-fed mice (Suppl. Figure 1C), indicating the development of hyperlipidemia. Glucose tolerance tests further showed that plasma glucose levels in the fructose-fed mice were significantly higher at 30, 60, 90 and 120 minutes compared to the chow-fed mice after glucose injection (Suppl. Figure 1D), suggesting the development of glucose intolerance in these mice. To investigate the impact of high fructose feeding on hepatic miRNA expression, miRNAs were extracted from livers and subjected to miRNA expression profiling by illumine-based deep sequencing. 303 mature miRNAs were detected through small non-coding RNA sequencing analysis. Among them, expression profiles of 45 miRNAs were found to be significantly different $(\mathrm{P}<0.05)$ between fructose feeding and chow diet. Of these 45 miRNAs, 19 were upregulated and 26 were downregulated with fructose feeding. A heat map was constructed to visualize the changes of these miRNAs (Figure 1A). Relative expression of these miRNAs in the livers of chow and fructose-fed mice is presented in Figure 1B. These results indicate that aberrant expression of miRNA profiles may be associated with the development of hyperlipidemia and insulin resistance in the fructose-fed mice.

\subsection{Fructose induced-miRNAs are extensively involved in lipid and carbohydrate metabolic pathways}


Bioinformatics analysis showed that ten out of the forty five miRNAs were assembled as a regulatory network that collaboratively targets multiple metabolic signaling molecules involved in lipid and lipoprotein metabolism [ e.g., SREBP-1c/2, ChREBP, insulin induced gene-1 (Insig1), gene-2 (Insig-2), and apolipoprotein B (apoB)] and insulin signaling (e.g., IRS-1 and FOXO1) (Figure 2) (Table 2A \& 2B). The following miRNAs were downregulated: miR-223-3p, miR-33-5p, miR-145a-3p, miR-125b-5p and miR-128-3p and the following miRNAs were upregulated: miR-19b-3p, miR-101a-3p -3p, miR-30a-5p, miR-378a-3p and miR-582-3p (Table $2 \mathrm{~A} \& 2 \mathrm{~B})$. Computing prediction further revealed that the potential target genes of these miRNAs included IRS-1 and FOXO-1 that mediate insulin signaling, SREBP-1c, SREBP-2, ChREBP, Insig-1 and Insig-2 (insig-2a and -2b) that are associated with hepatic de novo lipid synthesis as well as MTTP and apoB that are involved in VLDL assembly and secretion [10-13]. A schematic elucidating the network of interactions between these miRNAs, their potential target genes and the pathways involved is presented in Figure 2 (Solid lines represent connections between miRNAs and potential target genes and broken lines represent associations between miRNAs and metabolic pathways). This network indicated that one miRNA can be involved in multiple pathways whereas one gene can be a common target of several miRNAs that were induced by fructose feeding. For example, miR-223-3p potentially regulates mRNA expression of IRS-1, Insig-1 and apoB, and may be involved in pathways associated with insulin resistance and NAFLD (Table 2A). However, at the same time, IRS-1 is also a common target of miR-33-5p, miR-128-3p, miR-145a-3p, miR101a-3p, miR-125b-5p and miR-30-5p (Table 2A \& 2B). Detailed association between the aberrantly expressed miRNAs induced by high fructose feeding, their potential target genes and the pathways are described in Table $2 \mathrm{~A} \& 2 \mathrm{~B}$. This intriguing finding further stresses the unique and complex regulatory nature of miRNAs on cellular activity. It is now well accepted that an individual miRNA has the ability to regulate several groups of genes within a signaling pathway whereas multiple miRNAs can assemble as a network to 
collectively target a key gene in a signaling pathway [4]. This action significantly amplifies the regulatory effects of miRNAs in diverse cellular and physiological activities [4]. 
Table 2A. Fructose-downregulated miRNAs, their key target genes and the associated pathways

\begin{tabular}{|c|c|c|}
\hline MiRNAs & Target mRNA & Pathways \\
\hline $\mathrm{miR}-223-3 p$ & IRS-1, Insig-1, ApoB & $\begin{array}{l}\text { Insulin signaling, insulin resistance, fatty acid, } \\
\text { triglyceride and lipoprotein biosynthesis and } \\
\text { NAFLD. }\end{array}$ \\
\hline miR-33-5p & IRS-1, ChREBP, ApoB & $\begin{array}{l}\text { Insulin signaling, Insulin resistance, fatty acid, } \\
\text { triglyceride and lipoprotein biosynthesis and } \\
\text { NAFLD. }\end{array}$ \\
\hline miR-128-3p & IRS-1, FOXO1, MTTP & $\begin{array}{l}\text { Insulin signaling, insulin resistance, fatty acid, } \\
\text { triglyceride and lipoprotein biosynthesis and } \\
\text { NAFLD. }\end{array}$ \\
\hline $\operatorname{miR}-125 b-5 p$ & $\begin{array}{l}\text { IRS-1, SREBP-1c, } \\
\text { SREBP-2, Insig-1, Insig- } \\
\text { 2, ChREBP, ApoB }\end{array}$ & $\begin{array}{l}\text { Insulin signaling, insulin resistance, fatty acid, } \\
\text { triglyceride, lipoprotein and cholesterol } \\
\text { biosynthesis and NAFLD. }\end{array}$ \\
\hline miR-145a-3p & $\begin{array}{l}\text { IRS-1, FOXO1, MTTP, } \\
\text { ChREBP, ApoB }\end{array}$ & $\begin{array}{l}\text { Insulin signaling, insulin resistance, fatty acid, } \\
\text { triglyceride and lipoprotein biosynthesis and } \\
\text { NAFLD. }\end{array}$ \\
\hline
\end{tabular}


Table 2B. Fructose-upregulated miRNAs, their key target genes and the associated pathways

\begin{tabular}{|c|c|c|}
\hline MiRNAs & Target mRNA & Pathways \\
\hline miR-101a-3p & $\begin{array}{l}\text { IRS-1, SREBP-2, Insig- } \\
\text { 2, MTTP, АpoB }\end{array}$ & $\begin{array}{l}\text { Insulin signaling, Insulin resistance, fatty acid } \\
\text { and triglyceride, lipoprotein, cholesterol } \\
\text { biosynthesis and NAFLD. }\end{array}$ \\
\hline miR-30a-5p & $\begin{array}{l}\text { IRS-1, Insig-2, MTTP, } \\
\text { ApoB }\end{array}$ & $\begin{array}{l}\text { Insulin signaling, insulin resistance, Fatty acid } \\
\text { and TG, lipoprotein biosynthesis and NAFLD. }\end{array}$ \\
\hline miR-378a-3p & $\begin{array}{l}\text { SREBP-1c, ChREBP, } \\
\text { ApoB }\end{array}$ & $\begin{array}{l}\text { Insulin signaling, insulin resistance, Fatty acid } \\
\text { and TG, lipoprotein biosynthesis and NAFLD. }\end{array}$ \\
\hline miR-19b-3p & $\begin{array}{lr}\text { FOXO1, } & \text { SREBP-1c, } \\
\text { SREBP-2, } & \text { Insig-1, } \\
\text { ChREBP } & \end{array}$ & $\begin{array}{l}\text { Insulin signaling, insulin resistance, Fatty acid } \\
\text { and TG, lipoprotein, cholesterol biosynthesis } \\
\text { and NAFLD. }\end{array}$ \\
\hline miR-582-3p & FOXO1, ApoB & $\begin{array}{l}\text { Insulin signaling, insulin resistance and } \\
\text { lipoprotein biosynthesis. }\end{array}$ \\
\hline
\end{tabular}




\subsection{The association between fructose-induced miRNAs and their putative target genes}

To determine whether expression of the potential target genes was altered with fructose feeding, we measured the mRNA levels of IRS-1, FOXO1, SREBP-1c/2, ChREBP (Mlxipl), Insig-1, Insig-2, MTTP and apoB in the livers of chow and fructose-fed mice by q-RT-PCR. Expression of IRS-1 and Insig-2 (Insig-2a and Insig-2b) mRNAs was significantly reduced whereas mRNAs of FOXO1, SREBP-1c, SREBP-2, ChREBP, MTTP and apoB were significantly increased in the fructose-fed mice compared to chow-fed mice (Figures 3A -D). The expression profiles of these mRNAs inversely correlated with the abundances of their predicted miRNAs, suggesting that aberrant expressions of these miRNAs may, at least partially, contribute to the abnormal mRNA expression of these genes (Figures 1A, 3A-D). For instance, downregulation of miR-223-3p, miR-33-5p, miR-145a-3p, miR-125b-5p and miR-128-3p by fructose feeding may account for the increased mRNA abundance of FOXO1, ChREBP, Insig-1, MTTP and apoB due to the decreased interaction between these miRNAs and their target mRNAs (Figures 3A-D and Table 2A). On the other hand, upregulation of miR-101a-3p, miR-30a-5p and miR-378a-3p seems to target mRNAs of IRS-1 and Insig-2 which resulted in decreased mRNA expression of these two genes (Figures 3A-D and Table 2B).

Expression of the miR-378 family, including miR-378a, b, c and d, and miR-125a/b was either significantly upregulated or downregulated upon fructose feeding (Figures $1 \mathrm{~A}$ and B). To specifically determine the relationship between miRNAs and their potential target genes, we manipulated the expression of miR-378a and miR-125b-3p via genetic intervention or in vitro overexpression. We overexpressed a plasmid vector encompassed miR-125b-5p in McA cell and determined mRNA expression of MTTP and apoB by qRT-PCR. As shown in Figure 3E, mRNA abundance of MTTP was significantly reduced in the miR-125b-5p overexpressing cells compared to mock transfected cells (Figure 3E). Presence of miR-125b-5p further prevented palmitate (PA) induced overexpression of apoB mRNA (Figure 3F). Next, we subjected the wild type (WT) and genetic depletion of miR-378a mice (miR-378a-KO) to the high fructose diet and 
found that depletion of miR-378a protected the mice from fructose induced reduction of PI3K and IRS-1 (Figure 3G). Interestingly, we found that the abundance of miR-19b-3p and miR-582-3p were not inversely associated with their target mRNAs. Upregulation of these two miRNAs was found to be associated with increased mRNA levels of their target genes, such as FOXO1, SREBP-1c/2, ChREBP, Insig-1 and apoB (Figures 3A-D and Table 2B). At a glance, this is at odds with the widely accepted view of miRNA function to downregulate their target genes. However, recent studies have found that in addition to target mRNA degradation. miRNAs can also upregulate mRNA expression. The mRNA expression could be activated by the direct action of micro-ribonucleoproteins (miRNPs) and/or indirectly relieved from miRNA-mediated repression by abrogating the action of repressive miRNPs [14]. Taken together, our study identifies a set of metabolic genes which are common targets of the miRNAs induced by fructose feeding. Our data further implies that an individual miRNA has the potential to either downregulate or upregulate its targeted mRNAs simultaneously whereas an individual mRNA can be targeted by multiple miRNAs. This finding is in agreement with the recently identified mechanisms of action of miRNAs.

\subsection{Identification of novel physiological function for the fructose induced miRNAs.}

In an attempt to identify additional novel functions of the ten miRNAs that are altered by fructose feeding, we conducted intensive literature research for the reported physiological activities of these miRNAs. We found that miR-33-5p, also known as miR-33, is a wellcharacterized miRNA that is profoundly involved in hepatic triglyceride and cholesterol metabolism through targeting SREBP-1c gene expression [19]. In the current study, we found that expression of miR-33 was inversely associated with the mRNA abundance of ChREBP (Figures 1A and 3B), a major transcription factor involved in de novo lipogenesis in response to high carbohydrate diet [1], which may suggest that miR-33 could also regulate ChREBP in fructose metabolism. The reduced miR-33 in the fructose-fed mice may be responsible, at least partially, for the activation of ChREBP and the subsequent hyperlipidemia induced by high 
fructose feeding. Identification of ChREBP as a potential target of miR-33 broadens the targeting profile of miR-33 and may provide rationale for using this miRNA to treat carbohydrate induced hyperlipidemia. Moreover, we found that miR-101a-3p, miR-30a-5p and miR-582-3p have been reported to be involved in various cellular activities other than energy metabolic signaling. For example, expression of miR-101 is associated with skeletal muscle cell proliferation and differentiation [15] and can protect cardiac fibroblasts against hypoxia induced apoptosis [16]. Cellular abundances of miR-30a-5p and miR-582-3p are implicated in the development of breast cancer [17] and bladder cancer [18]. Thus, the current study would be the first to reveal that these three miRNAs are also involved in regulating lipid and carbohydrate metabolism. Our study further identified that miR-145a-3p, whose biological relevance has not been defined yet, may play a role in fructose-induced metabolic disorders because its expression profile was significantly downregulated by fructose (Figure 1A and 1B). Taken together, our study revealed that, in addition to their roles in cancer development and cell proliferation, miR-101a-3p, miR30a-5p, miR-582-3p and miRNA-145a-3p are involved in lipid and carbohydrate metabolism and may contribute to the onset of metabolic syndrome induced by high fructose feeding.

\subsection{High fructose diet and HFD induce different miRNA expression profiles in the livers.}

To explore whether the fructose-induced miRNA profile differs from that by HFD, we searched for published miRNA data sets that may have similar experimental design as ours but used high fat feeding instead of high fructose. Conducting vigorous literature research in the GEO database (http://www.ncbi.nlm.nih.gov/geo/) and PubMed, we found very little literature on this topic. We then used the miRNAs listed in Figure 1A as a starting point and searched for the expression patterns of each individual miRNA under high fat feeding condition for comparison. Due to the limited reports on high fat-induced miRNA profiles, we were unable to collect a complete profile match from HFD. Expression patterns of the miRNAs with high fructose feeding diet (from current study) and HFD (from literature reports) are presented in Table 3. Interestingly, we 
noticed that the expression patterns of miRNAs induced by fructose differed from those induced by HFD (Table 3). MiR-99b-5p, miR-150-5p, miR-100-5p, miR-223-3p, and miR-128-3p that were downregulated by fructose were upregulated in the livers of high fat-fed rats or mice (Table 3) $[5,20]$. In contrast, miR-130a, miR-30a-5p, miR-203-3p, miR-574-5p and miR-20a-5p that were induced by high fructose feeding were reduced in the high fat-fed rats or mice (Table 3 ) [5, 21, 22]. This intriguing finding suggests that different molecular mechanisms are involved in the development of metabolic syndrome induced by these two lipogenic diets. 
Table 3. Hepatic miRNA expression profiles altered by high fructose (HFru) diet and HFD

\begin{tabular}{|c|c|c|c|c|}
\hline MiRNAs & HFru (current study) & HFD (other studies) & Species & Ref. \\
\hline miR-99b-5p & Down & Up & Rat liver & [5] \\
\hline miR-150-5p & Down & $\mathrm{Up}$ & Rat Liver & [5] \\
\hline miR-100-5p & Down & $\mathrm{Up}$ & Rat Liver & {$[5]$} \\
\hline miR-223-3p & Down & $\mathrm{Up}$ & Rat liver & [5] \\
\hline miR-128-3p & Down & $\mathrm{Up}$ & Mouse liver & [20] \\
\hline miR-33-5p & Down & Down & Mouse liver & [23] \\
\hline miR-130a & $\mathrm{Up}$ & Down & Rat Liver & [5] \\
\hline miR-30a-5p & Up & Down & Rat Liver & [5] \\
\hline miR-203-3p & $\mathrm{Up}$ & Down & Rat Liver & [5] \\
\hline miR-574-5p & Up & Down & $\begin{array}{c}\text { Hepatocyte } \\
\text { cell line }\end{array}$ & [21] \\
\hline miR-20a-5p & Up & Down & Mouse liver & [22] \\
\hline miR-34a-5p & $\mathrm{Up}$ & $\mathrm{Up}$ & Mouse liver & [24] \\
\hline miR-378a-3p & Up & $\mathrm{Up}$ & Mouse liver & [25] \\
\hline miR-125b-5p & Down & - & - & - \\
\hline miR-145a-3p & Down & - & - & - \\
\hline miR-101a-3p & $\mathrm{Up}$ & - & - & - \\
\hline miR-19b-3p & $\mathrm{Up}$ & - & - & - \\
\hline miR-582-3p & $\mathrm{Up}$ & - & - & - \\
\hline
\end{tabular}




\section{DISCUSSION}

\subsection{Fructose-induced miRNAs in hepatic insulin signaling}

In this study, we found that IRS-1 was inhibited by fructose feeding which could be a consequence of the increased expression of miR-30a-5p, miR-101a-3p, miR-378a-3p and/or the decreased expression of miR-145-3p, miR-33, miR-223-3p, miR-125b-5p and miR-128-3p as all of these miRNAs content seed sequences to interact with the 3'-UTR of IRS-1 mRNAs. IRS-1 mediates insulin signaling by relaying the signal from the insulin receptor to downstream signaling molecules that regulate glucose and lipid metabolism [13]. Regulation of IRS1 by miR128a has been reported previously to be involved in myoblast proliferation and myotube hypertrophy in skeletal muscle [26]. Determination of miR-128-3p, along with other miRNAs stated above, in regulating expression of IRS-1 mRNA in the livers of fructose-fed mice provides novel mechanistic insight into the fructose-induced metabolic disorders. The observation that IRS-1 could be a common target of multiple miRNAs further strengthens the concept that multiple miRNAs can assemble as a regulatory network to repeatedly target an individual mRNA and generate synergistic impact on the expression of the target gene [4].

In this study, we observed that the mRNA abundance of FOXO1 was elevated in the livers of fructose-fed mice. FOXO1 belongs to the family of forkhead transcription factors involved in several cellular processes, including cell division, DNA repair, apoptosis, insulin signaling and lipid metabolism [27]. This observation is in accordance with a previous study which showed that protein levels of FOXO1 were increased by high-fructose feeding in hamsters [28]. Bioinformatics prediction further revealed that FOXO1 could be a potential common target of miR-19b-3p, miR-145-3p, miR-128-3p and miR-582-3p. It is very likely that the decreased levels of miR-145 and/or miR-128 caused by fructose feeding eases their inhibitory effects on FOXO1 mRNA expression, thus increasing FOXO1 expression with fructose feeding and contributing to the onset of diet-induced hyperglycemia. Regulation of FOXO1 by miRNAs highlights the 
complexity and fine balance between diverse cellular signaling in maintaining metabolic homeostasis.

\subsection{Fructose-induced miRNAs in hepatic lipid and lipoprotein metabolism}

The development of hyperlipidemia is closely associated with the development of T2D [1]. Hepatic de novo lipid synthesis is controlled by SREBPs, which induce expression of genes involved in fatty acid, triglyceride and cholesterol synthesis [11]. Activation of SREBPs is regulated by three ER membrane-bound proteins - Insig-1, Insig-2 and SCAP protein [29]. Low cellular sterol triggers the dissociation of the SCAP-SREBP complex from Insig-1 and Insig-2 thus allowing SCAP-SREBP complex to move to the Golgi apparatus, where the SREBPs are activated by proteolytic cleavage [29]. Hyperlipidemia is one of the major pathological phenotypes observed in high fructose-induced insulin resistant rodent models. However, the underlying mechanisms are not fully understood. Our study suggests that reduced expression of Insig-2 may derepress the inhibitory effect of Insig-2 on the precursors of SREBPs and enhance activation of SREBP-1c and -2, which may result in hyperactivation of SREBPs, leading to systemic hyperlipidemia in mice. As such, activation of SREBP-1c by high fructose diet may induce Insig-1 mRNA expression which could subsequently exert an inhibitory effect on SREBP1c activation by sequestering the proteins in the ER. However, the increased expression of Insig-1 apparently was insufficient to completely hinder the activation of SREBPs. The increased lipid substrates along with elevated levels of apoB and MTTP might favor the assembly and secretion of VLDL from hepatocytes and contribute to the hyperlipidemia induced by fructose.

In summary, our study identified a novel set of miRNAs that are abnormally expressed in the livers of fructose-fed mice. These miRNAs assemble as a regulatory network that cooperatively targets lipid metabolic pathways and insulin signaling at multiple levels to amplify its regulatory effect on the target genes and contribute to pathogenesis of fructose-induced insulin resistance. The miRNA profile induced by high fructose feeding differs from that of HFD, which implicates 
the different metabolic mechanisms in high fructose and high fat induced metabolic disorders. This study provides novel insight into the molecular mechanism of pathogenesis of high fructose diet induced metabolic syndrome and may provide rationale for pharmaceutical design to manipulate the expression of miRNAs in the prevention and treatment of high carbohydrate induced metabolic syndrome.

ACKNOWLEDGMENTS This work were supported by an NIH grant P20 GM104320-01A and Hatch funds from USDA/NIFA to Q. Su

\section{CONFLICT OF INTEREST}

The authors declare that they have no conflicts of interest with the contents of this article. 


\section{References}

[1] Dekker MJ, Su Q, Baker C, Rutledge AC, Adeli K. Fructose: a highly lipogenic nutrient implicated in insulin resistance, hepatic steatosis, and the metabolic syndrome. American Journal of Physiology-Endocrinology and Metabolism. 2010;299:E685-E94.

[2] Crapo PA, Kolterman OG, Henry RR. Metabolic consequence of two-week fructose feeding in diabetic subjects. Diabetes Care. 1986;9:111-9.

[3] Fabian MR, Sonenberg N, Filipowicz W. Regulation of mRNA translation and stability by microRNAs. Annual review of biochemistry. 2010;79:351-79.

[4] Wilczynska A, Bushell M. The complexity of miRNA-mediated repression. Cell Death \& Differentiation. 2015;22:22-33.

[5] Karolina DS, Armugam A, Tavintharan S, Wong MT, Lim SC, Sum CF, et al. MicroRNA 144 impairs insulin signaling by inhibiting the expression of insulin receptor substrate 1 in type 2 diabetes mellitus. PloS one. 2011;6:e22839.

[6] Sun Z, Evans J, Bhagwate A, Middha S, Bockol M, Yan H, et al. CAP-miRSeq: a comprehensive analysis pipeline for microRNA sequencing data. BMC genomics. 2014;15:1.

[7] Robinson MD, McCarthy DJ, Smyth GK. edgeR: a Bioconductor package for differential expression analysis of digital gene expression data. Bioinformatics. 2010;26:139-40.

[8] Chou C-H, Chang N-W, Shrestha S, Hsu S-D, Lin Y-L, Lee W-H, et al. miRTarBase 2016: updates to the experimentally validated miRNA-target interactions database. Nucleic acids research. 2016;44:D239-D47.

[9] Shannon P, Markiel A, Ozier O, Baliga NS, Wang JT, Ramage D, et al. Cytoscape: a software environment for integrated models of biomolecular interaction networks. Genome research. 2003;13:2498-504.

[10] Altomonte J, Richter A, Harbaran S, Suriawinata J, Nakae J, Thung SN, et al. Inhibition of Foxo1 function is associated with improved fasting glycemia in diabetic mice. American Journal of Physiology-Endocrinology and Metabolism. 2003;285:E718E28.

[11] Horton JD, Goldstein JL, Brown MS. SREBPs: activators of the complete program of cholesterol and fatty acid synthesis in the liver. The Journal of clinical investigation. 2002;109:1125-31.

[12] Koo H-Y, Wallig MA, Chung BH, Nara TY, Cho BS, Nakamura MT. Dietary fructose induces a wide range of genes with distinct shift in carbohydrate and lipid 
metabolism in fed and fasted rat liver. Biochimica et Biophysica Acta (BBA)-Molecular Basis of Disease. 2008;1782:341-8.

[13] Taniguchi CM, Ueki K, Kahn CR. Complementary roles of IRS-1 and IRS-2 in the hepatic regulation of metabolism. The Journal of clinical investigation. 2005;115:718-27.

[14] Valinezhad Orang A, Safaralizadeh R, Kazemzadeh-Bavili M. Mechanisms of miRNA-mediated gene regulation from common downregulation to mRNA-specific upregulation. International journal of genomics. 2014:970607.doi: 10.1155/2014/970607. [15] Li D, Zhan S, Wang Y, Wang L, Zhong T, Li L, et al. Role of microRNA-101a in the regulation of goat skeletal muscle satellite cell proliferation and differentiation. Gene. 2015;572:198-204.

[16] Zhao X, Wang K, Hu F, Qian C, Guan H, Feng K, et al. MicroRNA-101 protects cardiac fibroblasts from hypoxia-induced apoptosis via inhibition of the TGF- $\beta$ signaling pathway. The international journal of biochemistry \& cell biology. 2015;65:155-64.

[17] Li W, Liu C, Zhao C, Zhai L, Lv S. Downregulation of $\beta 3$ integrin by miR-30a-5p modulates cell adhesion and invasion by interrupting Erk/Ets - 1 network in triplenegative breast cancer. International journal of oncology. 2016;48:1155-64.

[18] Uchino K, Takeshita F, Takahashi R-u, Kosaka N, Fujiwara K, Naruoka H, et al. Therapeutic effects of microRNA-582-5p and-3p on the inhibition of bladder cancer progression. Molecular Therapy. 2013;21:610-9.

[19] Horie T, Nishino T, Baba O, Kuwabara Y, Nakao T, Nishiga M, et al. MicroRNA-33 regulates sterol regulatory element-binding protein 1 expression in mice. Nature communications. 2013;4.

[20] Povero D, Panera N, Eguchi A, Johnson CD, Papouchado BG, de Araujo Horcel L, et al. Lipid-induced hepatocyte-derived extracellular vesicles regulate hepatic stellate cells via microRNA targeting peroxisome proliferator-activated receptor- $\gamma$. CMGH Cellular and Molecular Gastroenterology and Hepatology. 2015;1:646-63. e4.

[21] Miyamoto Y, Mauer AS, Kumar S, Mott JL, Malhi H. Mmu-miR-615-3p regulates lipoapoptosis by inhibiting C/EBP homologous protein. PloS one. 2014;9:e109637.

[22] Tessitore A, Cicciarelli G, Del Vecchio F, Gaggiano A, Verzella D, Fischietti M, et al. MicroRNA expression analysis in high fat diet-induced NAFLD-NASH-HCC progression: study on C57BL/6J mice. BMC cancer. 2016;16:1.

[23] Rayner KJ, Suárez Y, Dávalos A, Parathath S, Fitzgerald ML, Tamehiro N, et al. MiR-33 contributes to the regulation of cholesterol homeostasis. science. 2010;328:15703 . 
[24] Xiao J, Bei Y, Liu J, Dimitrova-Shumkovska J, Kuang D, Zhou Q, et al. miR-212 downregulation contributes to the protective effect of exercise against non-alcoholic fatty liver via targeting FGF-21. Journal of cellular and molecular medicine. 2016;20:204-16.

[25] Liang T, Liu C, Ye Z. Deep sequencing of small RNA repertoires in mice reveals metabolic disorders-associated hepatic miRNAs. PloS one. 2013;8:e80774.

[26] Motohashi N, Alexander MS, Shimizu-Motohashi Y, Myers JA, Kawahara G, Kunkel LM. Regulation of IRS1/Akt insulin signaling by microRNA-128a during myogenesis. J Cell Sci. 2013;126:2678-91.

[27] Ponugoti B, Dong G, Graves DT. Role of forkhead transcription factors in diabetesinduced oxidative stress. Experimental diabetes research. 2012;2012.

[28] Qu S, Su D, Altomonte J, Kamagate A, He J, Perdomo G, et al. PPAR $\alpha$ mediates the hypolipidemic action of fibrates by antagonizing FoxO1. American Journal of Physiology-Endocrinology and Metabolism. 2007;292:E421-E34.

[29] Yabe D, Komuro R, Liang G, Goldstein JL, Brown MS. Liver-specific mRNA for Insig-2 down-regulated by insulin: implications for fatty acid synthesis. Proceedings of the National Academy of Sciences. 2003;100:3155-60. 


\section{Figure legends}

Fig 1. Heat map depicting miRNA expression profile of chow and fructose fed mouse livers (p-value $<0.05$ from the exact test in edgeR). (A) Each column represents either chow or fructose fed mice and each row represents one miRNA. Green color indicates downregulation and red color indicates upregulation with respect to chow diet. The color scale illustrates miRNA expression levels with increased expression ranging from green to red. (B) Bar graph depicting fold change $\left(\log _{2}\right)$ in miRNA expression in fructose fed compared to chow fed liver. $\mathrm{N}=6 /$ group.

Fig 2. MiRNA-mRNA network: Schematic showing relationship between putative miRNA target genes analyzed by q-RT-PCR with the corresponding miRNA targeting them and the metabolic pathways associated with energy metabolic processes such as hepatic de novo lipogenesis, lipid and lipoprotein metabolism, insulin signaling and NAFLD. Solid lines represent connections between miRNAs and potential target genes. Red solid lines are for upregulated miRNAs and green solid lines for downregulated miRNAs. Broken lines represent associations between miRNAs and metabolic pathways. Purple broken lines: insulin signaling and insulin resistance, brown broken lines: lipoprotein biosynthesis, black broken: fatty acid and triglyceride biosynthesis and NAFLD and grey broken: cholesterol biosynthesis.

Fig 3. Relative expression of potential target mRNAs of differentially expressed miRNA using q-RT-PCR. RNA samples from chow and fructose fed mice ( $n=6 /$ group) were reverse transcribed and mRNA levels of the putative target genes were determined by q-RT-PCR. (A) FOXO1, IRS-1; (B) SREBP-1c, SREBP-2 and ChREBP; (C) Insig-1, Insig-2a and Insig-2b and (D) MTTP and apoB. (E and F) mRNA levels of MTTP (E) and apoB (F) in McA cells overexpressing miR-125b-5p or a mock vector untreated or treated with palmitate (PA) $(100 \mu \mathrm{M})$ for 24 h. (G) mRNA levels of PI3K and IRS-1 in the wild type (WT) or miR-378a-KO (KO) 
mice with chow or fructose diet. Data were normalized to 18 srRNA. Results are shown as mean \pm SEM, $\mathrm{n}=6$ /group. $* \mathrm{P}<0.05$ versus controls. 


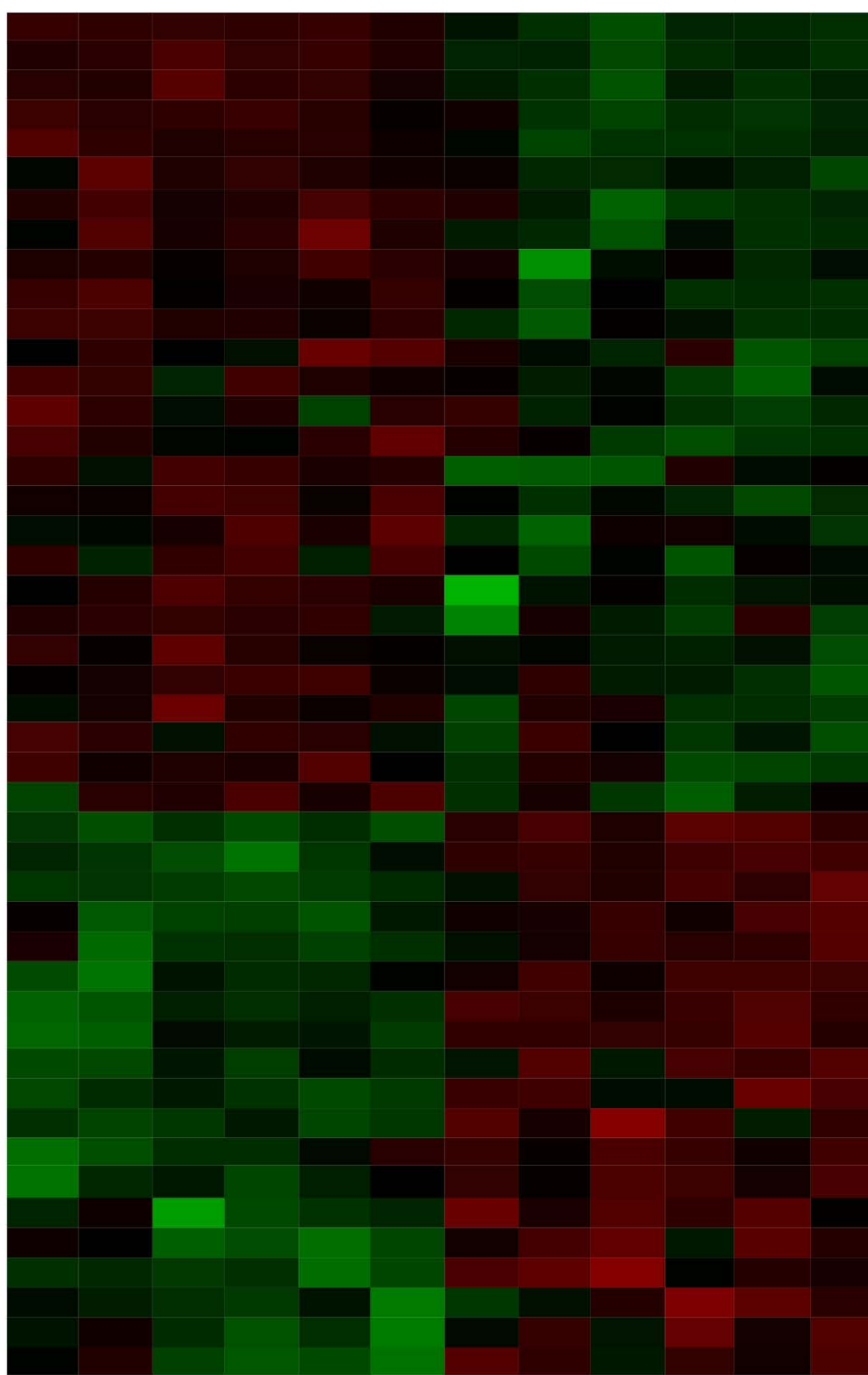

$m m u-m i R-100-5 p$ mmu-miR-125b-5p mmu-miR-99a-5p $m m u-m i R-501-3 p$ $m m u-m i R-99 b-5 p$ $m m u-m i R-150-5 p$ $m m u-m i R-145 a-3 p$ mmu-miR-126a-3p mmu-miR-22 $4-5 p$ mmu-miR-125a-5p $m m u-m i R-322-3 p$ mmu-miR-3105-3p $m m u-m i R-96-5 p$ mmu-miR-223-3p mmu-miR-122-3p mmu-miR-1247-5p mmu-miR-151-5p mmu-miR-676-3p $m m u-m i R-33-5 p$ mmu-miR-345-3p mmu-miR-1981-3p mmu-miR-674-3p mmu-miR-128-3p mmu-miR-1198-5p $m m u-m i R-212-5 p$ mmu-miR-191-3p $m m u-m i R-187-3 p$ mmu-miR-378d

mmu-miR-130a-3p $m m u-m i R-30 a-5 p$ $\mathrm{mmu}-\mathrm{miR}-101 \mathrm{c}$

$m m u-m i R-101 a-3 p$

mmu-miR-203-3p

$m m u-m i R-378 c$

$m m u-m i R-378 a-3 p$

mmu-miR-378a-5p

$m m u-m i R-378 b$

mmu-miR-674-5p

$\mathrm{mmu}-\mathrm{miR}-1843 \mathrm{~b}-5 \mathrm{p}$

$m m u-m i R-125 b-2-3 p$

$m m u-m i R-582-3 p$

$m m u-m i R-34 a-5 p$

mmu-miR-574-5p

mmu-miR-101a-5p

$m m u-m i R-20 a-5 p$

mmu-miR-19b-3p
mmu-miR-582-3p mmu-miR-674-5p mmu-miR-34a-5p mmu-miR-203-3p mmu-miR-378b

mmu-miR-378d mmu-miR-574-5p mmu-miR-101a-5p mmu-miR-378c mmu-miR-101c mmu-miR-30a-5p mmu-miR-378a-5p mmu-miR-1843b-5p

mmu-miR-20a-5p mmu-miR-125b-2-3p mmu-miR-130a-3p mmu-miR-378a-3p mmu-miR-19b-3p mmu-miR-101a-3p mmu-miR-126a-3p mmu-miR-151-5p mmu-miR-122-3p mmu-miR-1198-5p mmu-miR-676-3p mmu-miR-3105-3p mmu-miR-223-3p mmu-miR-1981-3p mmu-miR-33-5p mmu-miR-191-3p mmu-miR-322-3p mmu-miR-345-3p mmu-miR-125a-5p mmu-miR-150-5p mmu-miR-128-3p mmu-miR-674-3p mmu-miR-99a-5p mmu-miR-125b-5p mmu-miR-145a-3p mmu-miR-1247-5p mmu-miR-99b-5p mmu-miR-96-5p mmu-miR-212-5p mmu-miR-100-5p mmu-miR-224-5p mmu-miR-501-3p

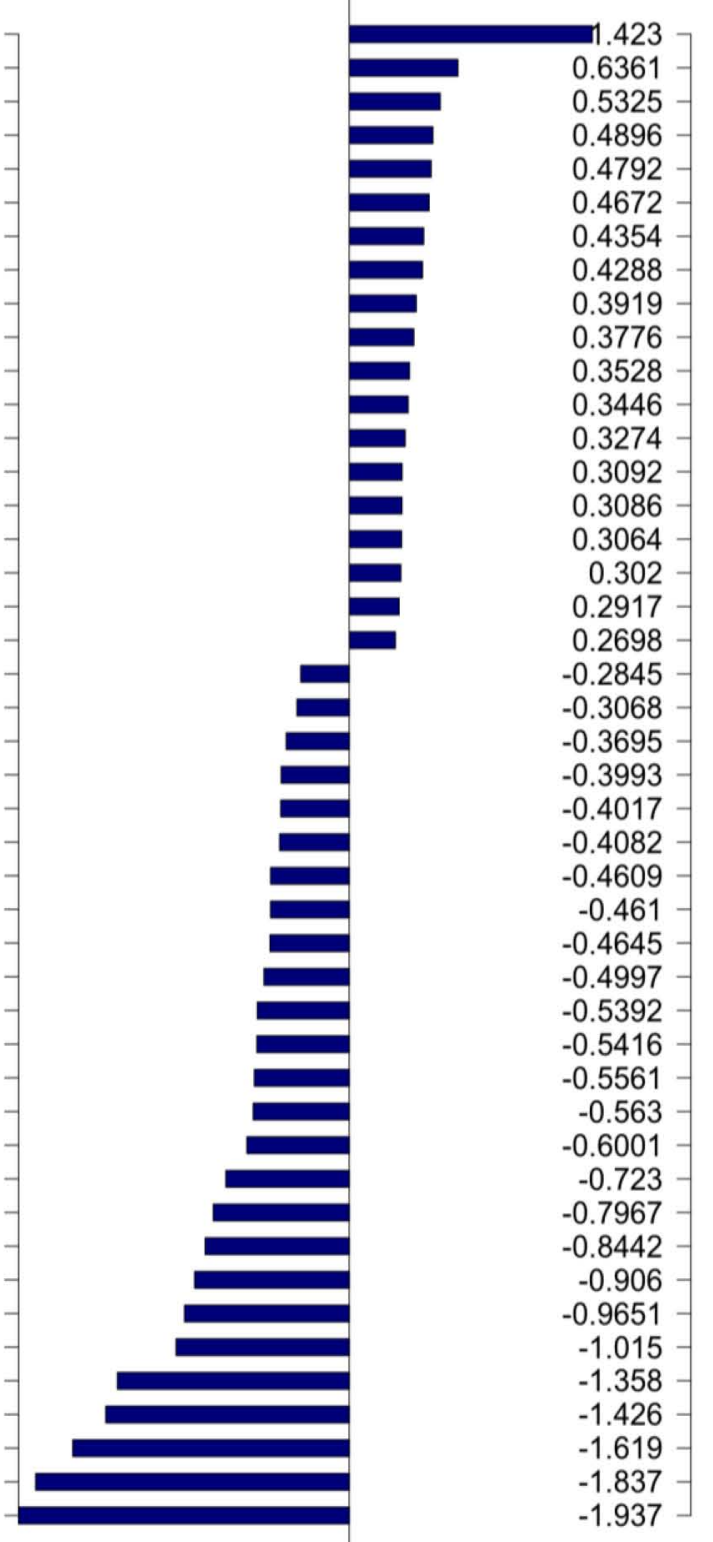


